\title{
Computer Simulation of Grain Growth Kinetics with Solute Drag
}

\author{
Danan Fan \\ P.O. Box 5800, MS 1411, Sandia National Laboratories, Albuquerque, New \\ Mexico 87185

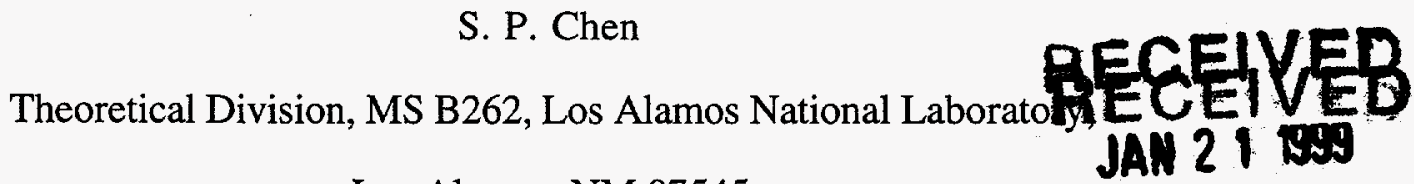 \\ Los Alamos, NM 87545 \\ Long-Qing Chen

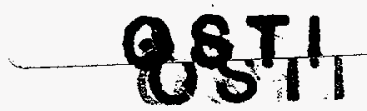 \\ Department of Materials Science and Engineering, The Pennsylvania State \\ University, University Park, PA 16862
}

\begin{abstract}
The effects of solute drag on grain growth kinetics were studied in two dimensional (2-D) computer simulations by using a diffuse-interface field model. It is shown that, in the low velocity / low driving force regime, the velocity of a grain boundary motion departs from a linear relation with driving force (curvature) with solute drag. The nonlinear relation of migration velocity and driving force comes from the dependence of grain boundary energy and width on the curvature. The growth exponent $m$ of power growth law for a polycrystalline system is affected by the segregation of solutes to grain boundaries. With the solute drag, the growth exponent $\mathrm{m}$ can take any value between 2 and 3 depending on the ratio of lattice diffusion to grain boundary mobility. The grain size and topological distributions are unaffected by solute drag, which are the same as those in a pure system.
\end{abstract}




\section{DISCLAIMER}

This report was prepared as an account of work sponsored by an agency of the United States Government. Neither the United States Government nor any agency thereof, nor any of their employees, make any warranty, express or implied, or assumes any legal liability or responsibility for the accuracy, completeness, or usefulness of any information, apparatus, product, or process disclosed, or represents that its use would not infringe privately owned rights. Reference herein to any specific commercial product, process, or service by trade name, trademark, manufacturer, or otherwise does not necessarily constitute or imply its endorsement, recommendation, or favoring by the United States Government or any agency thereof. The views and opinions of authors expressed herein do not necessarily state or reflect those of the United States Government or any agency thereof. 


\section{DISCLAIMER}

Portions of this document may be illegible in electronic image products. Images are produced from the best available original document. 


\section{Introduction}

Grain growth is a process of grain boundary migration to decrease total grain boundary area and total free energy of a system, driven by mean curvatures of grain boundaries. The kinetics of grain growth depends strongly on the presence or absence of solute or impurity segregation at grain boundaries. In a pure material, the only process which occurs during grain growth is local atomic rearrangement. If solute segregation is present, the migration of grain boundaries may be controlled by long range diffusion. It is well understood that grain growth follows power growth law $R_{t}^{m}-R_{0}^{m}=k t$ with the growth exponent $\mathrm{m}=2$ in a pure material $1,2,3$, where $R_{0}$ is the initial average grain size, $R_{t}$ is the average grain size at time $\mathrm{t}$ and $\mathrm{k}$ is the kinetic coefficient. Experimentally, however, the growth exponent $\mathrm{m}$ is found to be larger than 2 even if a very low impurity level (a few ppm or less) is present in pure metals $1,4,5$.

Theoretically, Cahn ${ }^{6}$ studied the effects of solute drag on migration kinetics of grain boundaries by considering the interaction of impurity atmosphere with grain boundaries. Cahn predicted that, according to the migration velocity and driving force, the migration of grain boundaries with impurity segregation can be classified into different regimes: (a) a low velocity / low driving force regime, where long-range diffusion of impurity is important; (b) a high velocity / high driving regime, where long-range diffusion is not necessary and desorption of solute may occur according to the diffusivity of impurity; and (c) a transition region between these two regimes. It was also shown that the migration velocity of grain boundaries may not be linearly proportional to the driving force if impurity is segregated to grain boundaries, which depends on the migration velocity, driving force and the concentration of segregation. Hillert and Sundman 7 obtained similar results to those of Cahn by using a free energy dissipation theorem and idealized models for interaction energy profiles. Even though the theory explains a number of experimental observations, the direct derivation of a grain-growth law from this theory is difficult. 
Krzanowski and Allen 8, 9, 10 studied the effects of segregation on antiphase boundary migration kinetics by employing a diffuse-interface theory. They obtained a relationship of migration velocity with driving force (here it is the mean curvature of curved boundaries) in the low velocity / low driving force regime, which is found to be equivalent to that of Cahn ${ }^{9}$. They indicated that the value of the interfacial mobility is dependent on the presence of segregation and the kinetics of boundary migration is retarded when the segregation of solute is present at boundaries. Interestingly, however, they showed that, by assuming interfacial energy and interfacial thickness are constant, the growth exponent $\mathrm{m}(=2)$ is unaffected by the presence of segregation at boundaries 8,9 . Their experimental results of $\mathrm{Fe}-\mathrm{Al}$ alloys seem to support their theoretical predictions 10 . Krzanowski and Allen 11 also found both theoretically and experimentally that, however, the growth exponent $\mathrm{m}$ will change to 3 if the antiphase domain boundaries are wetted by a thin layer of a second phase, indicating that long-range diffusion controls the coarsening kinetics in this case. Despite theoretical prediction of the nonlinear relation between the migration velocity and the driving force in the presence of solute drag, other researchers predicted that the solubility of impurity may also affect the growth exponent $m^{12}$, i.e., $m$ is 3 for low solubility of impurity and is 2 in high solubility regime. Therefore, from the theoretical point of view, it is still unclear if and how the solute drag will affect the growth exponent $m$ for polycrystalline materials.

Recently, the authors have developed a diffuse-interface field model for simulating the grain growth in pure materials 13,14 and for studying the microstructure evolution in volume-conserved two-phase systems 15,16. A significant feature of this model is that the microstructural complexity and long-range diffusion can be taken into account conveniently and simultaneously. Computer simulations using this model allow one not only to monitor the detailed temporal microstructure evolution during grain growth but also to obtain all the information about the average grain size and size distributions. In this paper, we modify 
this diffuse-interface field model to study the effects of solute drag on grain growth kinetics. We focus on the grain growth in the low velocity / low driving force regime, in which the driving force for grain boundary migration is the mean curvature (capillarity) and solute atmosphere moves with grain boundaries. The migration of grain boundaries with solute drag was studied by computer simulations and the effects of segregation on growth exponent were analyzed for polycrystalline materials.

\section{Diffuse-Interface Field Model}

We assume a binary alloy consisting of elements $\mathrm{X}$ and $\mathrm{Y}$ for studying grain growth in a solid solution with solute drag. At a certain temperature $\mathrm{T}$, there are two possible stable solid solutions $\alpha$ and $\beta$ with the equilibrium concentrations $C_{\alpha}$ and $C_{\beta}$ respectively in this system. By choosing the average alloy concentration $\bar{C}$ within the solid solution regions ( $\bar{C} \leq \mathrm{C}_{\alpha}$ or $\bar{C} \geq \mathrm{C}_{\beta}$ ), the grain growth with solute drag can be studied.

In this diffuse-interface field model, an arbitrary polycrystalline microstructure is described by a set of continuous field variables,

$$
\eta_{1}(r), \eta_{2}(r), \ldots \ldots, \eta_{p}(r)
$$

where $\mathrm{p}$ is the number of possible orientations in space and $\eta_{i}(\mathrm{i}=1, \ldots, \mathrm{p})$ are called orientation field variables which distinguish the different orientations of grains and are continuous in space, and $\mathrm{r}$ is the position in the space. Their values continuously vary from -1.0 to 1.0 . In real materials, the number of orientations is infinite $(p=\infty)$. However, it was shown that a finite number of $p(p>30)$ might be sufficient to realistically simulate grain growth $13-15$. 
To study the effects of solute segregation, a concentration field $C(r)$ is introduced, which describes the spatial distribution of solute atoms. Within the diffuse interface theory 17 , the total free energy of an inhomogeneous system can be written as:

$$
\begin{aligned}
F=\int[ & f_{o}\left(C(r) ; \eta_{1}(r), \eta_{2}(r), \ldots, \eta_{p}(r)\right) \\
& \left.+\frac{\kappa_{C}}{2}(\nabla C(r))^{2}+\sum_{i=1}^{p} \frac{\kappa_{i}}{2}\left(\nabla \eta_{i}(r)\right)^{2}\right] d^{3} r
\end{aligned}
$$

,where $\nabla C$ and $\nabla \eta_{i}$ are gradients of concentration and orientation fields, $\kappa_{C}$ and $\kappa_{i}$ are the corresponding gradient energy coefficients, and $f_{o}$ is the local free energy density which, in this work, is assumed to be,

$$
f_{o}=f_{1}(C)+\sum_{i=1}^{p} f_{2}\left(C, \eta_{i}\right)+\sum_{i=1}^{p} \sum_{j \neq i}^{p} f_{3}\left(\eta_{i}, \eta_{j}\right)
$$

in which

$$
\begin{gathered}
f_{1}(C)=-(A / 2)\left(C-C_{m}\right)^{2}+(B / 4)\left(C-C_{m}\right)^{4}+\left(D_{\alpha} / 4\right)\left(C-C_{\alpha}\right)^{4}+\left(D_{\beta} / 4\right)\left(C-C_{\beta}\right)^{4} \\
f_{2}\left(C, \eta_{i}\right)=-(\gamma / 2)\left[\left(C-C_{\alpha}\right)^{2}+\left(C-C_{\beta}\right)^{2}\right]\left(\eta_{i}\right)^{2}+(\delta / 4)\left(\eta_{i}\right)^{4} \\
f_{3}\left(\eta_{i}, \eta_{j}\right)=\left(\varepsilon_{i j} / 2\right)\left(\eta_{i}\right)^{2}\left(\eta_{j}\right)^{2}
\end{gathered}
$$

where $C_{\alpha}$ and $C_{\beta}$ are the solubilities $X$ or $Y$ in $\alpha$ and $\beta$ phases respectively, $C_{m}=\left(C_{\alpha}+\right.$ $\left.\mathrm{C}_{\beta}\right) / 2, \mathrm{~A}, \mathrm{~B}, \mathrm{D}_{\alpha}, \mathrm{D}_{\beta}, \gamma, \delta$, and $\varepsilon_{i j}$ are phenomenological parameters. A main requirement for $f_{o}$ is that it has $2 \mathrm{p}$ degenerate minima at equilibrium concentration $\mathrm{C}_{\alpha}$ or $\mathrm{C}_{\beta}$ to distinguish the $2 \mathrm{p}$ orientation differences of grains in space. The parameters are chosen in such a way that $f_{o}$ has $2 \mathrm{p}$ degenerate minima with equal depth located at $\left(\eta_{1}, \eta_{2}, \ldots, \eta_{p}\right)=$ $(1,0, \ldots, 0),(0,1, \ldots, 0), \ldots,(0,0, \ldots, 1)$ at the equilibrium concentration $C_{\alpha}$ or $C_{\beta}$. This requirement ensures that each point in space can only belong to a grain with a given 
orientation of a given phase. The justification for using such a free energy model in the study of coarsening was discussed previously 13-15.

The energy of a planar grain boundary, $\sigma_{g b}$, between a grain of orientation $i$ and another grain of orientation $\mathrm{j}$ for two stable $\alpha$ grains, can be written as follows,

$$
\sigma_{g b}=\int_{-\infty}^{+\infty}\left[\Delta f\left(\eta_{i}, \eta_{j}, C\right)+\frac{\kappa_{C}}{2}\left(\frac{d C}{d x}\right)^{2}+\frac{\kappa_{i}}{2}\left(\frac{d \eta_{i}}{d x}\right)^{2}+\frac{\kappa_{j}}{2}\left(\frac{d \eta_{j}}{d x}\right)^{2}\right] d x
$$

in which

$$
\Delta f\left(\eta_{i}, \eta_{j}, C\right)=f_{o}\left(\eta_{i}, \eta_{j}, C\right)-f_{o}\left(\eta_{i, e}, \eta_{j, e}, C_{\alpha}\right)-\left(C-C_{\alpha}\right)\left(\frac{\partial f_{o}}{\partial C}\right)_{\eta_{i, e}, \eta_{j, e}, C_{\alpha}}
$$

where $f_{o}\left(\eta_{i, e}, \eta_{j, e}, C_{\alpha}\right)$ represents the free energy density minimized with respect to $\eta_{i}$ and $\eta_{j}$ at the equilibrium composition of $\alpha$ phase $C_{\alpha}$. If average concentration $\bar{C} \geq$ $\mathrm{C}_{\beta}, C_{\alpha}$ is replaced by $C_{\beta}$ in equation (4) since $\beta$ phase is the only stable phase.

The evolution of orientation and concentration field variables are described by the time-dependent Ginzburg-Landau (TDGL) 18 and Cahn-Hilliard 19 equations,

$$
\begin{aligned}
& \frac{d \eta_{i}(r, t)}{d t}=-L_{i} \frac{\delta F}{\delta \eta_{i}(r, t)}, \quad \mathrm{i}=1,2, \ldots, \mathrm{p}, \\
& \frac{d C(r, t)}{d t}=\nabla\left\{D \nabla\left[\frac{\delta F}{\delta C(r, t)}\right]\right\}
\end{aligned}
$$

where $L_{i}$ and $D$ are kinetic coefficients related to the grain boundary mobilities and atomic diffusion coefficients, $t$ is time, and $F$ is the total free energy given in equation (1). The 
microstructural evolution of grain growth with solute drag can be studied by numerically solving eqn. $5 \mathrm{a}$ and $5 \mathrm{~b}$ coupled kinetic equations.

\section{Numerical methodology}

To numerically solve the set of kinetic equations (5), one needs to discretize them with respect to space. We discretize the Laplacian using the following approximation,

$$
\nabla^{2} \phi=\frac{1}{(\Delta x)^{2}}\left[\frac{1}{2} \sum_{j}\left(\phi_{j}-\phi_{i}\right)+\frac{1}{4} \sum_{j^{\prime}}\left(\phi_{j^{\prime}}-\phi_{i}\right)\right]
$$

where $\phi$ is any function, $\Delta x$ is the grid size, $j$ represents the set of first nearest neighbors of $i$, and $j^{\prime}$ is the set of second nearest neighbors of $i$. For discretization with respect time, we employed the following simple Euler technique,

$$
\phi(t+\Delta t)=\phi(t)+\frac{d \phi}{d t} \times \Delta t
$$

where $\Delta t$ is the time step for integration. All the results discussed below were obtained by using $\Delta \mathrm{x}=2.0, \Delta t=0.1$ to ensure numerical stability. The kinetic equations are discretized in 2 -D by using $512 \times 512$ square grid points with periodic boundary conditions applied along both directions. The total number of orientation field variables are $36(\mathrm{p}=$ 36).

The following parameters for the free energy function (eq. 2 ) were assumed in this study: $\mathrm{A}=2.0, \mathrm{~B}=9.88, \mathrm{C}_{\alpha}=0.05, \mathrm{C}_{\beta}=0.95, \mathrm{D}_{\alpha}=\mathrm{D}_{\beta}=1.52, \gamma=2.0, \delta=1.0, \varepsilon_{i j}=$ 3.0, which give two equilibrium solid solutions and satisfy the requirements mentioned in section 2 for free energy function $f_{o}$. We also assumed isotropic grain boundary energies $\kappa_{i}=\kappa_{j}=\kappa=2.0$ and $\kappa_{C}=2.0$. The mobilities are chosen as $L_{1}=L_{2}=L=1.0$, which give isotropic grain boundary mobility. In this study, the average concentration of the alloy 
was chosen as $\bar{C}=0.04$, which is less than the solubility of $\alpha$ phase $\left(\bar{C}<\mathrm{C}_{\alpha}\right)$ and hence ensures that the microstructure consists of single phase $\alpha$ grains with the segregation of $Y$ atoms at grain boundaries.

To generate the initial microstructure, a single phase grain growth simulation was first performed to obtain a fine grain structure. Grains are then randomly assigned with the average concentration $\bar{C}$ and an orientation field. The concentration of solute atoms is conserved during the simulations of grain growth, e.g., average concentration $\bar{C}$ is a constant and solute atoms can only diffuse within the system. The initial segregation was obtained by relaxing the system for certain time steps (100 time steps), which gives a grain structure with solute segregation at grain boundaries. The kinetics of grain growth with solute drag was then studied. The area of each grain at a given time step is directly calculated from the microstructure by counting the number of grid points within a grain and grain size $\mathrm{R}$ is obtained from the area by assuming a circular shape for all grains, therefore, Area $=\pi R^{2}$. The average grain radius at a given time step is then obtained by averaging over all the grains in a system. All the kinetic data and size distributions were obtained using $512 \times 512$ grid points and averaged from independent runs. There are more than 3000 grains at the beginning of collecting data for calculating the statistics and there are about 200 at the end.

\section{Migration of A Grain Boundary with Solute drag}

To study the effects of solute drag on the migration of a grain boundary, we consider a circular grain $\left(\eta_{1}\right)$ embedded in another grain $\left(\eta_{2}\right)$. We employed $200 \times 200$ square lattice points to spatially discretize the kinetic equations with periodic boundary conditions applied along both Cartesian coordinate axes. The initial radius of the circular grain was chosen to have 60 grid points. 
The microstructural evolution of the circular grain is shown in Fig. 1. It can be seen from Fig. 1 that the grain boundary is very smooth and circular at all times, indicating that the parameters chosen for numerical simulations are proper and no apparent lattice anisotropy is introduced by discretizing the continuous equations (5). The snap shots of concentration profiles across the middle of the circular grain are shown in Fig. 2. The segregation of solutes at grain boundary is obvious and the solute atmosphere moves with grain boundary, which indicates a low velocity / low driving force condition (case (a)). It should be noted that, in this dynamic system, the velocity of grain boundary is not constant and there is no time for the concentration profile to relax to its equilibrium shape at a given time and velocity. Therefore, many runs are needed to calculated the average segregation concentration at a given time step.

The driving force for the grain boundary movement is the mean curvature, which is $1 / R$ in this case ( $R$ is the radius of the circular grain). It was shown that, if without solute drag, the kinetics of a circular grain follows the equation $R_{0}^{2}-R^{2}=2 L \kappa t 18,20,21$, where $R_{0}$ is the original radius of the circular grain, $R$ is the radius at time t, gradient coefficient $\kappa_{1}=\kappa_{2}=\kappa$ and the mobility $L_{1}=L_{2}=L$. In this case, the area of the circular grain decreases linearly with respect to time t. However, the migration of grain boundary with solute drag may not follow this linear relationship. To study the effects of kinetic conditions on grain boundary migration kinetics, two kinetic conditions are employed. First, the ratio between the kinetic coefficient $D$, which is related to the solute diffusivity in lattice, and the kinetic coefficient $\mathrm{L}$, which is related to grain boundary mobility, is chosen to be 1.0. Under this condition, the lattice diffusion rate is fast enough so that solute atoms can move with grain boundaries without significantly delaying the grain boundary motion. Second, the ratio of kinetic coefficients $D$ and $L$ is chosen to be 0.5 by keeping $L$ fixed at its value for case one and decreasing the kinetic coefficient $\mathrm{D}$. The time evolution of the areas of the circular grain with solute drag is shown in Fig. 3. It can be seen that areas of the circular grain decrease slightly nonlinearly with time for $\mathrm{D} / \mathrm{L}=1.0$ conditions. The 
smaller the $\mathrm{D} / \mathrm{L}$ ratio, the more obvious the nonlinearity. When the diffusion of solute atoms in the lattice is fast enough ( condition $\mathrm{D} / \mathrm{L}=1.0$ ), long-range lattice diffusion of solute atoms has less effect on grain boundary migration. In this case, the relation between grain areas and time is very close to a linear one (Fig. 3). However, if the rate of lattice diffusion of solute is much smaller than the motion of grain boundaries ( condition $\mathrm{D} / \mathrm{L}=$ $0.5)$, long-range diffusion becomes the controlling factor for grain boundary migration and the shrinkage kinetics of the circular grain departs from the linear relation.

For the single phase grain growth (without solute drag), the velocity of grain boundary migration can be expressed as $18,20,21$ :

$$
v=M\left(\mathrm{~K}_{1}+\mathrm{K}_{2}\right)
$$

where $\mathrm{v}$ is the velocity, $\left(\mathrm{K}_{1}+\mathrm{K}_{2}\right)$ is the mean curvature of the grain boundary, and $\mathrm{M}$ is a kinetic constant dependent on coefficients $L$ and $\kappa$. For a 2-D circular grain, $v=d R / d t$, $\left(\mathrm{K}_{1}+\mathrm{K}_{2}\right)=\cdots 1 / \mathrm{R}$, and equation (9) can be easily integrated into the relation $R_{0}^{2}-R^{2}=2 L \kappa t$, i.e., a linear relation between grain area and time t. However, the velocity expression obtained for grain growth with solute drag was given by 6,9 :

$$
v=\frac{\mu \sigma}{\delta}\left(\mathrm{K}_{1}+\mathrm{K}_{2}\right)
$$

where $\sigma$ is the grain boundary energy, $\delta$ is grain boundary thickness, and $\mu$ is a coefficient which is a function of diffusivity, surface excess energy and other thermodynamic factors. If we assume that $\sigma, \delta$ and $\mu$ are independent of the curvature (or grain radius $\mathrm{R}$ ), equation (10) can also be integrated into a similar relation $R_{0}^{2}-R^{2}=2 \mu \sigma t / \delta$ for a circular grain. Actually, Krzanowski and Allen 8,9 made the same assumptions and obtained the growth law with growth exponent $\mathrm{m}=2$ for the polycrystalline grain growth with solute drag. With these assumptions, the only difference 
between grain growth with and without solute drag is the kinetic coefficient of the growth law, while the growth exponent $m$ will not be affected.

However, Fig. 3 indicates that the velocity with solute drag is not linearly related to the driving force $\left(\left(\mathrm{K}_{1}+\mathrm{K}_{2}\right)\right.$ or $1 / \mathrm{R}$ for a circular grain). To directly visualize the relation of the velocity with the driving force for grain boundary migration, we plot $d R / d t$ with $1 / R$ (curvature) in Fig. 4 for the shrinkage of a circular grain in simulations. The $\mathrm{dR} / \mathrm{dt}$ values were obtained by fitting the R-t curve with a smooth function and then taking derivatives of that function at time $t$. The relation of $\mathrm{dR} / \mathrm{dt}$ and $1 / \mathrm{R}$ for gain boundary motion without solute drag is also plotted in Fig. 4 for comparison. Fig. 4 shows that the velocity of grain boundary migration without solute drag keeps a good linear relation with curvature (driving force), which ensures that the growth exponent $\mathrm{m}$ is 2 in the power law. On the other hand, as solute atoms segregate to grain boundaries, the velocity starts to depart from the linear relationship, which results in the change of growth exponent $\mathrm{m}$ and a nonlinear relation between the average grain area and time. It is also shown that the migration velocity is dramatically decreased by solute drag, i.e., at a certain driving force (curvature), the velocities with solute drag are much smaller than that without solute drag (Fig. 4). The smaller the diffusivity, the more severely the grain boundary motion will be retarded. This slowing-down actually confirms the Krzanowski and Allen's formulation ${ }^{9}$ in the low velocity / low driving force regime.

To investigate which parameter affects the linear relation of velocity and driving force, the dependence of grain boundary energy on curvature is plotted in Fig. 6. It can be seen that grain boundary energy $\sigma$ decreases as $1 / \mathrm{R}$ increases and there is no simple relation can be extracted from the simulation data. Since $\sigma$ decreases as curvature increases, the slope of the velocity - curvature curve will decrease as curvature increases ( equation (9) ), i.e., the $v-1 / \mathrm{R}$ relation will curve down when solute segregation occurs. This is what exactly observed in Fig. 4. 
The segregation concentration of solute at grain boundaries will affect the grain boundary energy 22 . Therefore, the variation of solute concentration with curvature is shown in Fig. 5. In this plot, the dots are measured maximum concentrations at moving grain boundaries and the solid line is a fit to the average of these data. It was reported 22 that a linear relation exists between grain boundary energy $\sigma$ and segregation concentration. However, there is no such linear relation can be extracted from current simulations. It can be seen that the segregation concentration increases with the increase of curvature $(1 / R)$ in current simulations. This result seems to show a different trend from that obtained by Cahn 6 . He showed that as grain boundaries speed up, they always desorb more and more solute. Therefore, one may expect that in simulations, as a circular grain shrinks, its velocity increases and there should be a smaller amount of solute at smaller grain size.

We believe that this difference comes from the fact that, in Cahn's work, the concentration profile at a boundary is only influenced by the part of the boundary still approaching and it reflects no influence of the parts already past ${ }^{6}$. As a result, more and more solute is left behind the boundary as it speeds up. However, in the current model, the desorption of solute from the moving boundary increases the concentration of solute in the bulk of grain past, which drives the bulk of grain away from its thermodynamic equilibrium and significantly increases the total free energy of the system. To minimize the total free energy, the solute is pushed back to the grain boundary region by diffusion, which is only possible in the low velocity/low driving regime. The diving force for this process is the chemical potential difference in the supersaturated solid solution and in the boundary region. As the circular grain shrinks, there is less total grain boundary area in the system. Therefore, there are more solute segregated at the grain boundary as the system evolves, since we employed a conserved system. The significance of this process is that a much larger solute drag effect is imposed on grain boundaries than that predicted by Cahn. This raises an interesting point that the solute drag effect may be also dependent on the 
shape of the free energy curve as a function of concentration (i.e., the value of $\partial f_{o} / \partial c$ ) for the solid solution at a given temperature. If the change of the free energy curve with concentration is sharp, the solute is not likely to desorb from the grain boundary, which imposes a greater drag or pinning effect on grain boundaries. Of course, the desorption of the solute from boundaries occurs much more easily if this process does not affect the bulk free energy severely.

The grain boundary width (full width) may also change with curvature since the segregation concentration at grain boundary varies with curvature. The relation of calculated grain boundary width and curvature is shown in Fig. 7. It is shown that the grain boundary width increases with the increase of curvature. According to equation (9), the increase of grain boundary width with curvature will also make the $v-1 / R$ curve bend down. However, the effect of grain boundary width on migration velocity of a grain boundary may be important only if the grain size is compatible to grain boundary width, i.e., for nanocrystals. For a normal system in which grain size is much larger than the width of grain boundaries, the variation of grain boundary energy with curvature may be the dominant reason for the deviation of the linear relation between velocity and curvature. Therefore, it can be concluded that the migration velocity of grain boundaries with solute drag is not linearly proportional to the driving force. The nonlinear relation of migration velocity and driving force comes from the dependence of grain boundary energy and the width on the curvature, which may result in the change of growth exponent $m$ in the growth law.

\section{Effect of Solute drag on Grain Growth of Polycrystals}

With solute drag, the grain boundary motion will be retarded and the velocity will depart from linear relation with driving force. In this section, the effect of solute drag on 
power growth law, grain size distributions and topological distributions in 2-D polycrystalline systems were studied. We chose a $512 \times 512$ cell with 36 orientation field variables $(\mathrm{p}=36)$, which is sufficient to realistically simulate grain growth ${ }^{13-15}$.

A typical microstructural evolution of grain growth with solute drag is shown in Fig. 8. The characteristics of these microstructures are essentially similar to those of grain growth in a pure system. The only noticeable difference is that the life time of a quadrijunction ( a junction of four grains which is thermodynamically unstable in these systems ) with solute drag is longer than that in a pure system, which is resulted from the fact that microstructural evolution is controlled by diffusion in solute drag systems. Therefore, some quadrijunctions have been captured in microstructures at different time steps, which is seldom observed in simulation and experimental microstructures of a pure system 13 .

To study the kinetics of grain growth with solute drag, two kinetic conditions, $\mathrm{D} / \mathrm{L}=1.0$ and $\mathrm{D} / \mathrm{L}=0.5$, were chosen, which are the same as previous sections. The average grain radius as a function of time for the $D / L=1.0$ system is shown in Fig. 9. To extract growth exponent $\mathrm{m}$ and coefficient $\mathrm{k}$, the data were then fitted to the equation $\bar{R}_{t}^{m}-\bar{R}_{0}^{m}=k t$ by a multi-parameter nonlinear least-square fitting routine. The growth exponent $\mathrm{m}$ for this system is found to be $2.1 \pm 0.01$, which is very close to $\mathrm{m}=2$ in a pure system. In this system, the lattice diffusion rate is compatible to the diffusion rate across grain boundaries, therefore, long-range lattice diffusion is not the limiting factor for grain boundary motion. It is shown in above section that grain boundary velocity slightly departs from linear relation with driving force. As a result, the growth exponent $\mathrm{m}$ for this system is not significantly affected by the solute drag.

The time dependence of average grain size in the $D / L=0.5$ system is shown in Fig. 10. It can be seen that growth exponent $m$ has changed to $2.49 \pm 0.01$, significantly different from that in $\mathrm{D} / \mathrm{L}=1.0$ system. It is well understood that growth exponent $\mathrm{m}$ is 2 
for grain growth of a pure system $\mathrm{m} 1,2,13$, and it is 3 for Ostwald ripening process in two-phase systems, in which long-range diffusion controls coarsening kinetics 15 . Clearly, growth exponent $\mathrm{m}$ in grain growth with solute drag can not be easily determined, which is dependent on diffusion mechanisms in a system. In the $D / L=0.5$ system, lattice diffusion rate is not fast enough to follow the grain boundary migration and the motion of grain boundaries is pinned by solute atoms. Therefore, coarsening kinetics is controlled by lattice diffusion and growth exponent $\mathrm{m}$ departs significantly from that in a pure system.

It is clear that, according to the ratio of lattice diffusion and grain boundary mobility, long-range diffusion plays different roles in determining the growth law of a polycrystalline system. It can be expected that, if lattice diffusion is much slower than grain boundary migration, the growth exponent can be very close to 3 , in which grain boundary motion is totally controlled by lattice diffusion. Therefore, with solute drag, the growth exponent $\mathrm{m}$ can have a value anywhere between 2 and 3 depending on the ratio of lattice diffusion and grain boundary mobility. This result seems to explain a variety of growth exponents $m$ obtained experimentally from different alloys systems 1,4 , in which the growth exponent values vary from $m=2$ to $m=3$ with an average of $2.5 \pm 0.4$ and diffusion mechanisms vary significantly.

The time dependences of grain size distributions in $D / L=1.0$ and $D / L=0.5$ systems are shown in Fig. 11 and Fig. 12, respectively. For comparison, a typical grain size distribution obtained from 2-D simulations for a pure system (without solute drag) is also shown in Fig. 11. Two conclusions can be drawn from these two figures. First, both systems have reached a scaling or steady state, i.e., the shape of grain size distribution is time-invariant. Second, by comparing these figures, it can be seen that the shapes of grain size distributions are the same in these two systems, which are almost identical to that in a pure system (Fig. 11) ${ }^{13}$. The topological distributions in $D / L=1.0$ and $D / L=0.5$ systems are shown in Fig. 13 and Fig. 14 at different time steps. A topological distribution for a 
pure system is compared in Fig. 13. It is also clear that topological distributions are timeinvariant. Comparison of two systems shows that the shapes of topological distributions are identical to each other, indicating that topological distributions are unaffected by solute drag. The peak of topological distributions in both systems is located at 5-sided grains, which is identical to that for a pure system (Fig. 13) ${ }^{14}$ while the peak can change to 6sided grains in a two-phase system 16,20 . Therefore, even though the grain growth kinetics is very sensitive to solute drag and diffusion mechanisms, the grain size and topological distributions will not be altered by solute drag and diffusion mechanisms, which are the same as those in a system without solute drag.

\section{Conclusions}

Our computer simulations of grain boundary migration with solute drag show that

the motion of a grain boundary is greatly retarded by solute segregation at grain boundary. In the low velocity / low driving force regime, the velocity of a mobile grain boundary departs from a linear relation with driving force (curvature) with solute drag. The diffusivity and diffusion mechanisms will affect the migration velocity and grain growth kinetics. The nonlinear relation of migration velocity and driving force comes from the dependence of grain boundary energy and width on the curvature. We have shown that the growth exponent $m$ of power growth law for a polycrystalline system will be affected by the segregation of solute to grain boundaries. With the solute drag, the growth exponent $\mathrm{m}$ can take any value between 2 and 3 depending on the ratio of lattice diffusion and grain boundary mobility. It is found that the grain size and topological distributions are unaffected by solute drag and diffusion mechanisms, which are the same as those in a pure system. 
Acknowledgments: D. F. and S. P. C.'s work is supported by the U.S. Department of Energy, Division of Materials Science, Office of Basic Energy Science, and L-Q C.'s work is supported by the National Science Foundation under the grant number DMR 9633719 and the simulations were performed at the Pittsburgh Supercomputing Center and the Advanced Computing Laboratory at Los Alamos National Laboratory.

Sandia is a multiprogram laboratory operated by Sandia Corporation, a Lockheed Martin Company, for the United States Department of Energy under contract DE-AC04-94AL85000. 


\section{References}

(1) Atkinson, H. V., Acta Metall., 36, 469 (1988).

(2) Glazier, J. A., Philosophical Mag. B , 62, 615 (1990).

(3) Fradkov, V. E., Physica D: Nonlinear Phenomena, 66, 50 (1993).

(4) J. W. Martin and R. D. Doherty, In Stability of Microstructure in Metallic Systems, p. 228, Cambridge University Press (1976).

(5) M. Guttmann and D. Mclean, Interface Segregation (edited by W. C. Johnson and D. M. Blakely), pp. 261-350. Am. Soc. Metal, Metals Park, Ohio (1977).

(6) J. W. Cahn, Acta Metall, 10, 789 (1962).

(7) M. Hillert and B. Sundman, Acta Metall, 24, 731 (1976).

(8) J. E. Krzanowski and S. M. Allen, Surface Science, 144, 153 (1984).

(9) J. E. Krzanowski and S. M. Allen, Acta Metall., 34, 1035 (1986).

(10) J. E. Krzanowski and S. M. Allen, Acta Metall., 34, 1045 (1991).

(11) J. E. Krzanowski and S. M. Allen, Acta Metall., 31, 213 (1983).

(12) R. J. Brook, In Ceramic Fabrication Processes (Ed. by F. F. Y. Wang), p. 331. Academic Press, New York (1976).

(13) Danan Fan and L.-Q. Chen, Acta Mater., 45, 611-622 (1997); Danan Fan and L.-Q. Chen, Acta Mater., 45, 1115-1126(1997).

(14) L.-Q. Chen and W. Yang, Phys. Rev. B 50, 15752 (1994).

(15) Danan Fan and L.-Q. Chen, Acta Mater., 45, 3297-3310(1997).

(16) Danan Fan and L.-Q. Chen, J. Am. Ceram. Soc., 79, 1163 (1997).

(17) J. W. Cahn and J. E. Hilliard, J. Chem. Phys., 28 (1958) 258.

(18) S. M. Allen and J. W. Cahn, Acta Metall., 27, 1085 (1979).

(19) J. W. Cahn, Acta Metall., 9, 795 (1961).

(20) Danan Fan, Ph.D. dissertation, The Pennsylvania State University, 1996, p69-83.

(21) Danan Fan and L-Q Chen, Phil. Mag. Lett., 75, 187(1997).

(22) E. D. Hondros and M. P. Seah, Physical Metallurgy, 3rd ed., edited by R. W. Cahn and P. Haasen, p855, North-Holland, Amsterdam, (1983). 


\section{Figure Captions}

Fig. 1. The microstructural evolution of a circular grain.

Fig. 2. The time dependence of concentration profiles across the grain boundary of a circular grain. Dotted line: $t=1000$; double dotted line $t=3000$; solid line: $t=5000$.

Fig. 3. The time dependence of the grain area of a circular grain with kinetic condition $\mathrm{D} / \mathrm{L}=1.0$ and $\mathrm{D} / \mathrm{L}=0.5$.

Fig. 4. The relationship of driving force ( curvature $1 / R$ ) with the migration velocity of grain boundary in a pure system and in systems with segregation under kinetic conditions $\mathrm{D} / \mathrm{L}=0.5$ and $\mathrm{D} / \mathrm{M}=1.0$.

Fig. 5. The calculated relation of the peak concentration of segregation at grain boundary with the curvature of a circular grain.

Fig. 6 . The calculated dependence of grain boundary energy on the mean curvature of a circular grain.

Fig. 7. The calculated dependence of grain boundary width on the mean curvature of a circular grain.

Fig. 8. The microstructural evolution of grain growth with solute drag in a 2-D polycrystalline system. (a) $\mathrm{t}=5000$; (b) $\mathrm{t}=10000 ;$ (c) $\mathrm{t}=15000$; (d) $\mathrm{t}=20000$.

Fig. 9. The time dependence of average grain size under kinetic condition $D / L=1.0$. The solid line is a nonlinear fit to power growth law $\bar{R}_{t}^{m}-\bar{R}_{0}^{m}=k t$, which gives $\mathrm{m}=2.1 \pm 0.01$.

Fig. 10. The time dependence of average grain size under kinetic condition $D / L=0.5$. The solid line is a nonlinear fit to power growth law $\bar{R}_{t}^{m}-\bar{R}_{0}^{m}=k t$, which gives $\mathrm{m}=2.49 \pm 0.01$ 
Fig. 11 The time dependence of grain size distributions under kinetic condition $\mathrm{D} / \mathrm{L}=1.0$. The solid line is a typical grain size distribution in a pure system (without solute drag).

Fig. 12. The time dependence of grain size distributions under kinetic condition $\mathrm{D} / \mathrm{L}=0.5$.

Fig. 13, The time dependence of grain topological distributions under kinetic condition $\mathrm{D} / \mathrm{L}=1.0$. A topological distribution for a pure system (without solute drag) is shown in this figure for comparison.

Fig. 14, The time dependence of grain topological distributions under kinetic condition $\mathrm{D} / \mathrm{L}=0.5$. 


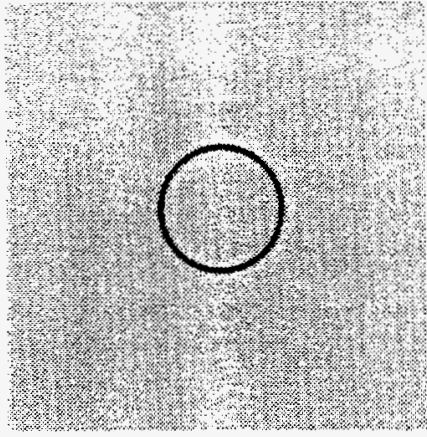

(a)

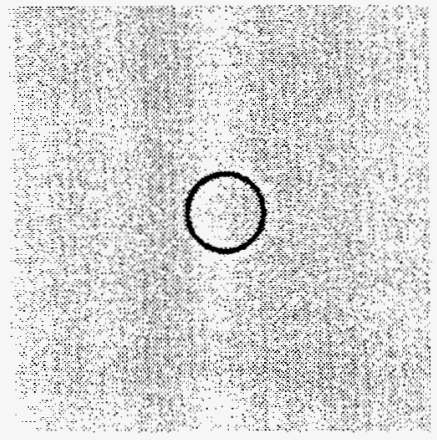

(c)

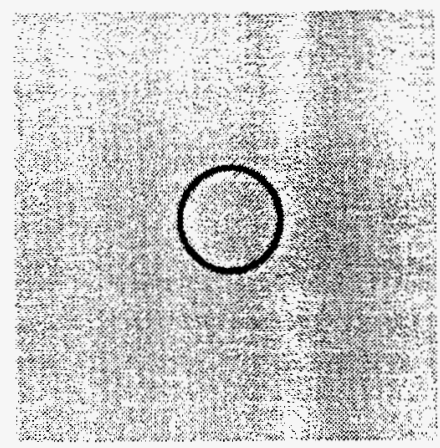

(b)

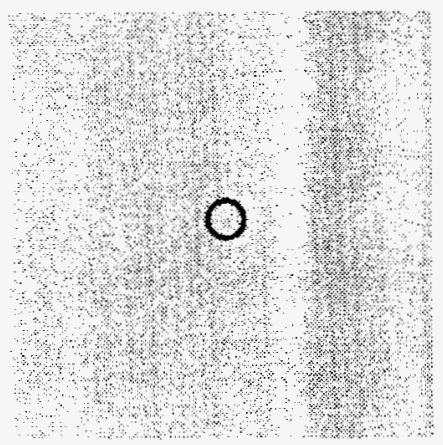

(d)

Fig. 1 The temporal evolution of a circular grain with solute-drag. (a) time step $=1000$;(b) time step $=3000$;(c) time step $=5000$; (d) time step $=7000$. 


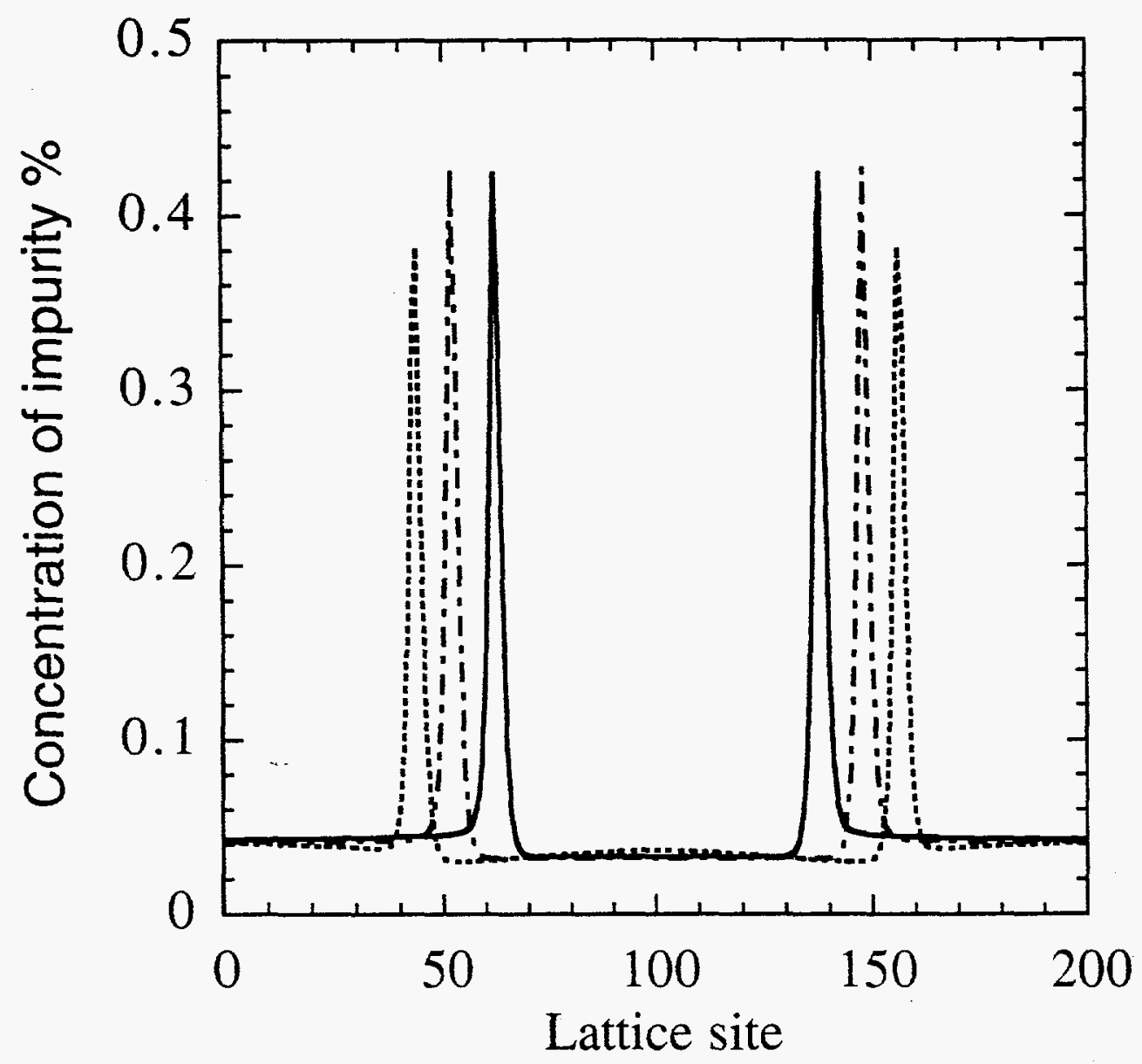

Fig. 2 The time dependence of concentration profiles across the grain boundary of a circular grain. Dotted line: $t=1000$; double dotted line $t=3000$; solid line: $\mathrm{t}=5000$. 


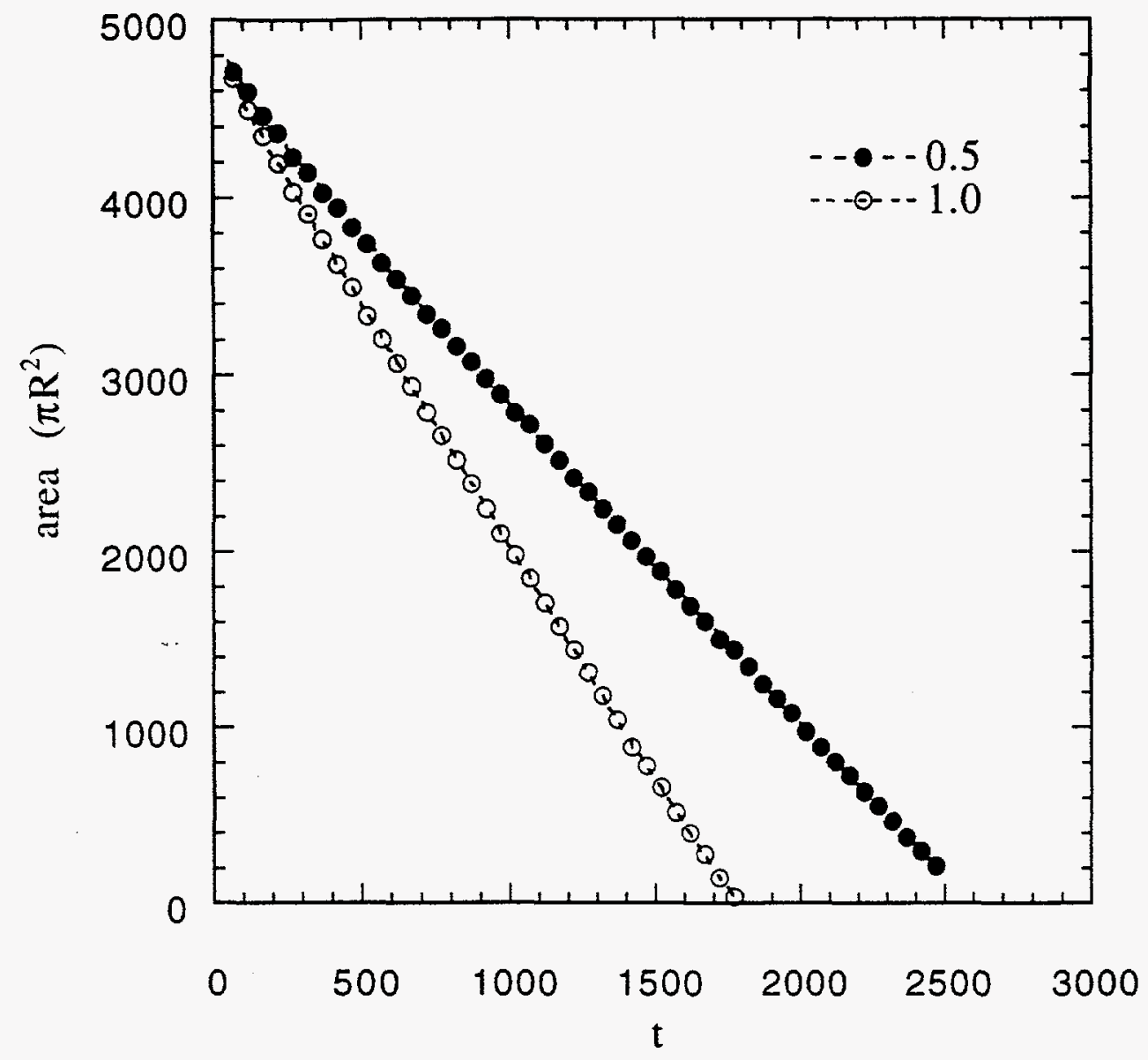

Fig. 3. The time dependence of the grain area of a circular grain with kinetic condition $\mathrm{D} / \mathrm{L}=1.0$ and $\mathrm{D} / \mathrm{L}=0.5$. 


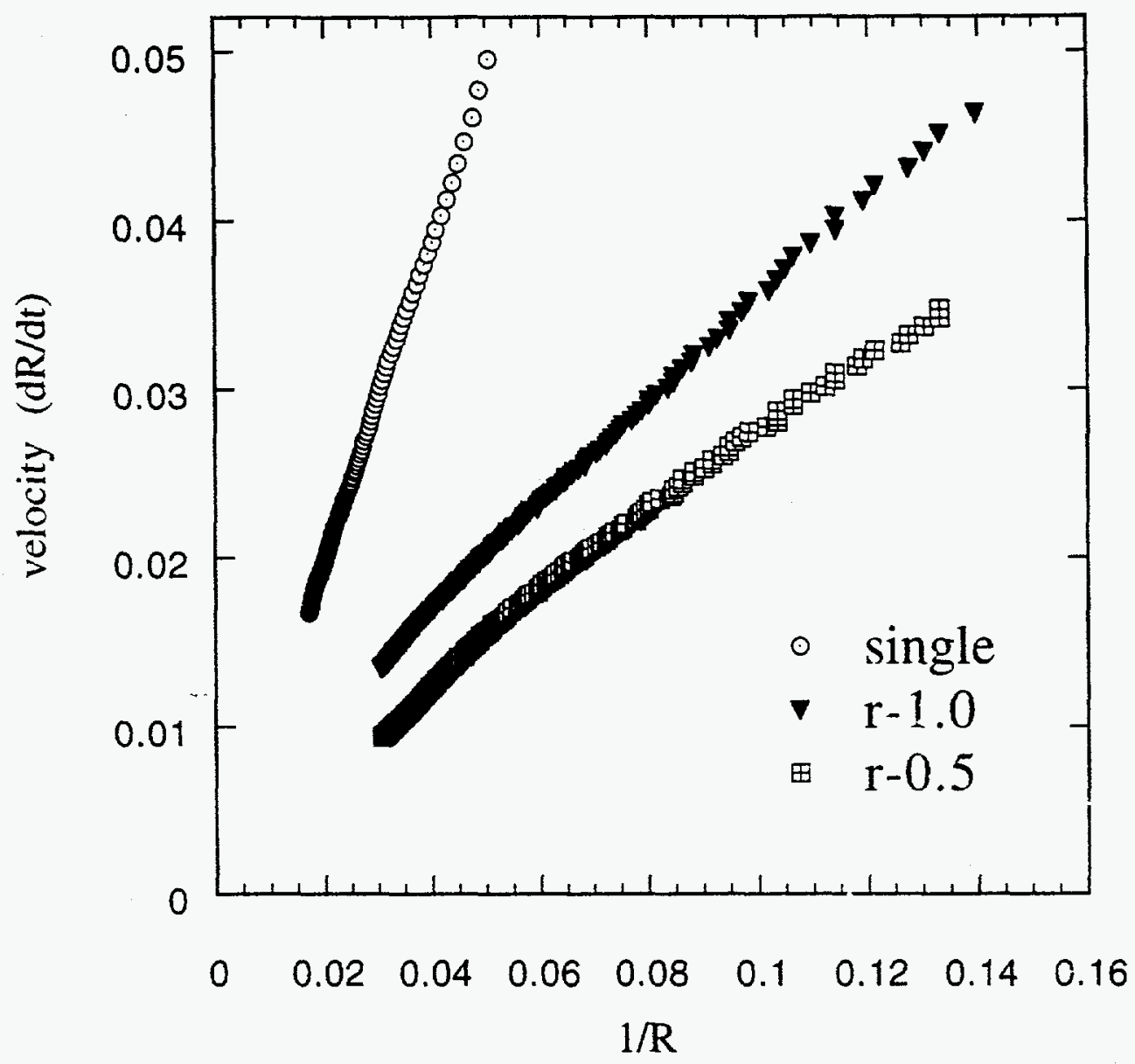

Fig. 4. The relationship of driving force (curvature $1 / \mathrm{R}$ ) with the migration velocity of grain boundary in a pure system and in systems with segregation under kinetic conditions $\mathrm{D} / \mathrm{L}=0.5$ and $\mathrm{D} / \mathrm{M}=1.0$. 


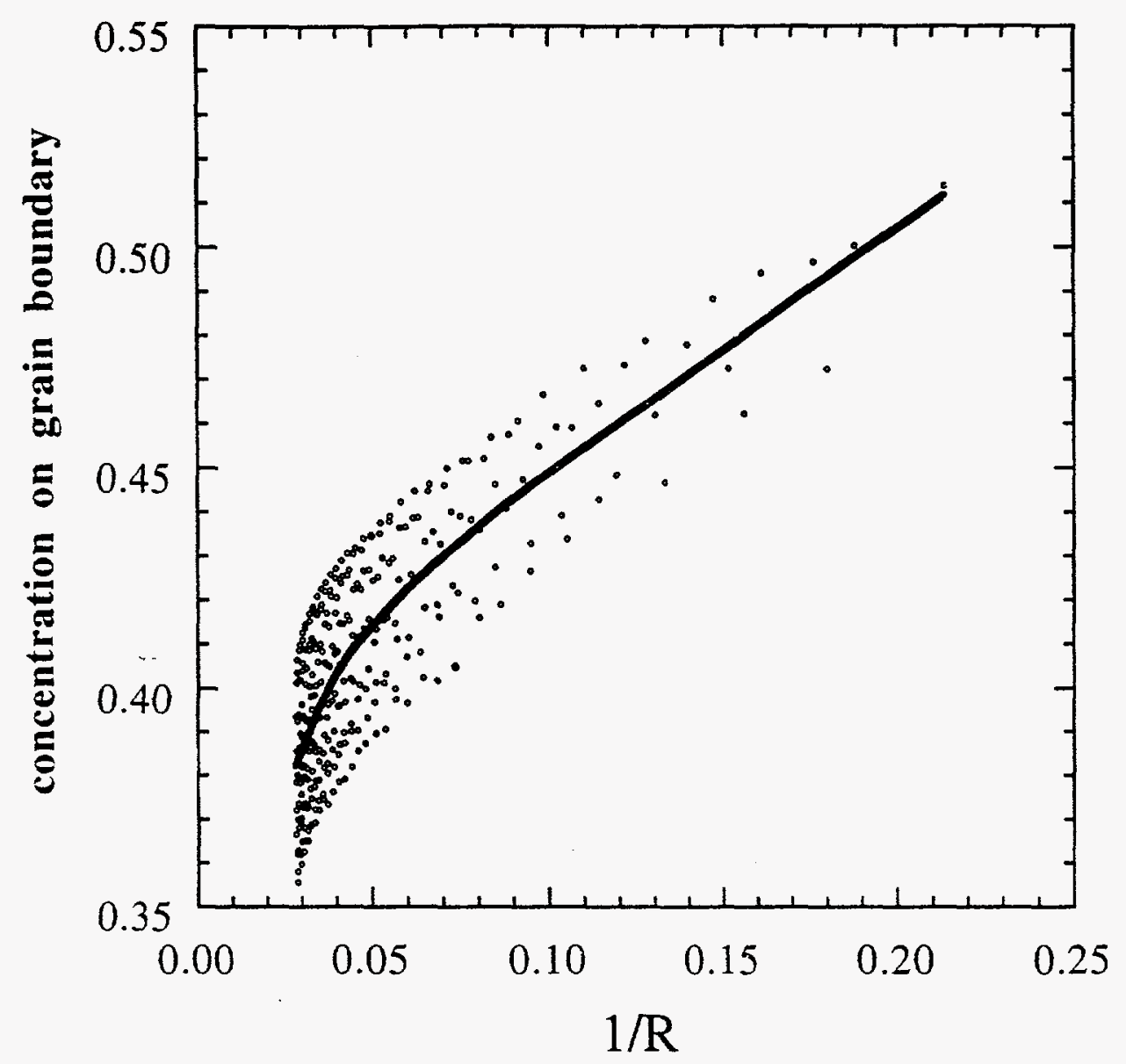

Fig. 5. The calculated relation of the peak concentration of segregation at grain boundary with the curvature of a circular grain. 


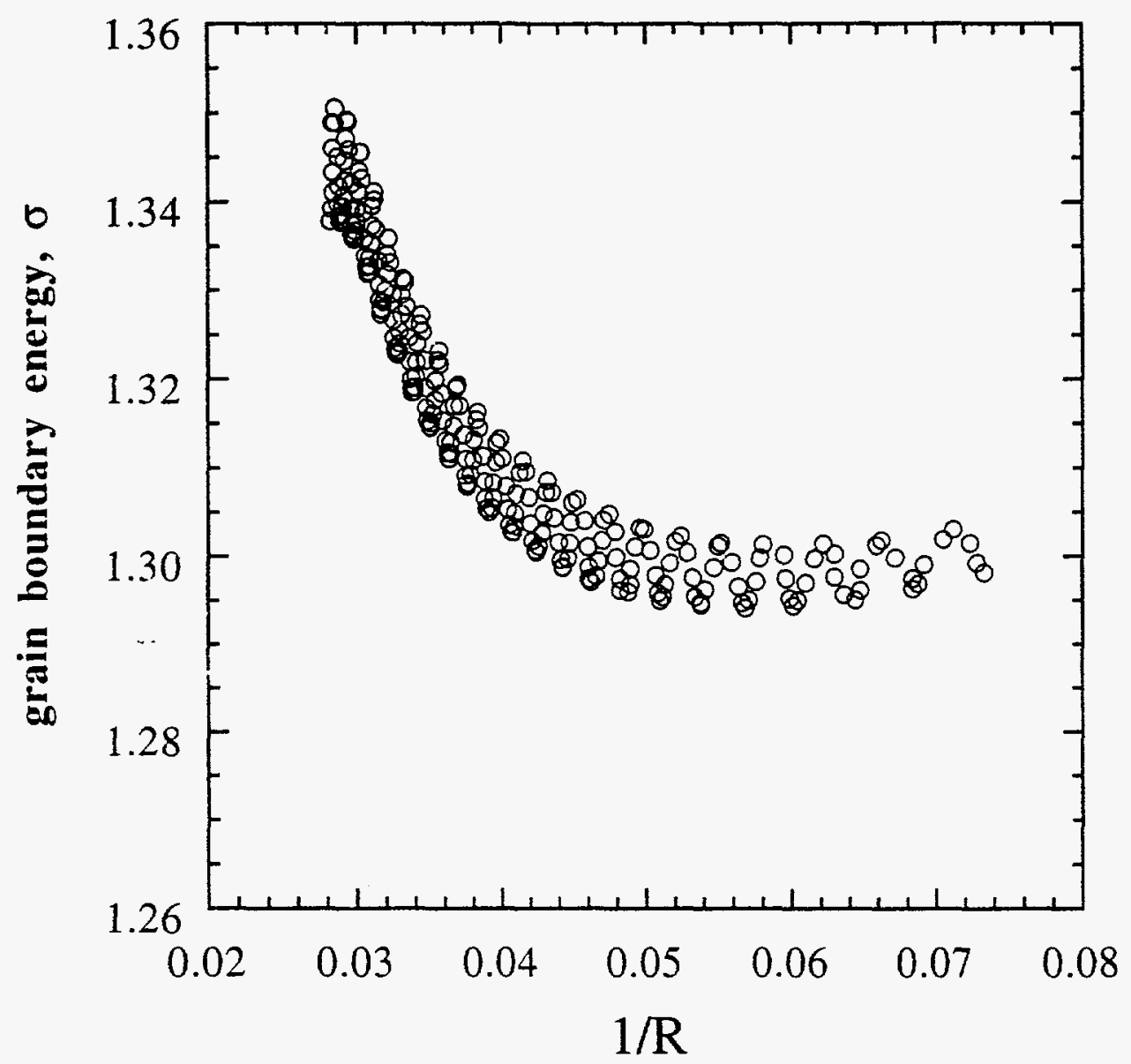

Fig. 6. The calculated dependence of grain boundary energy on the mean curvature of a circular grain. 


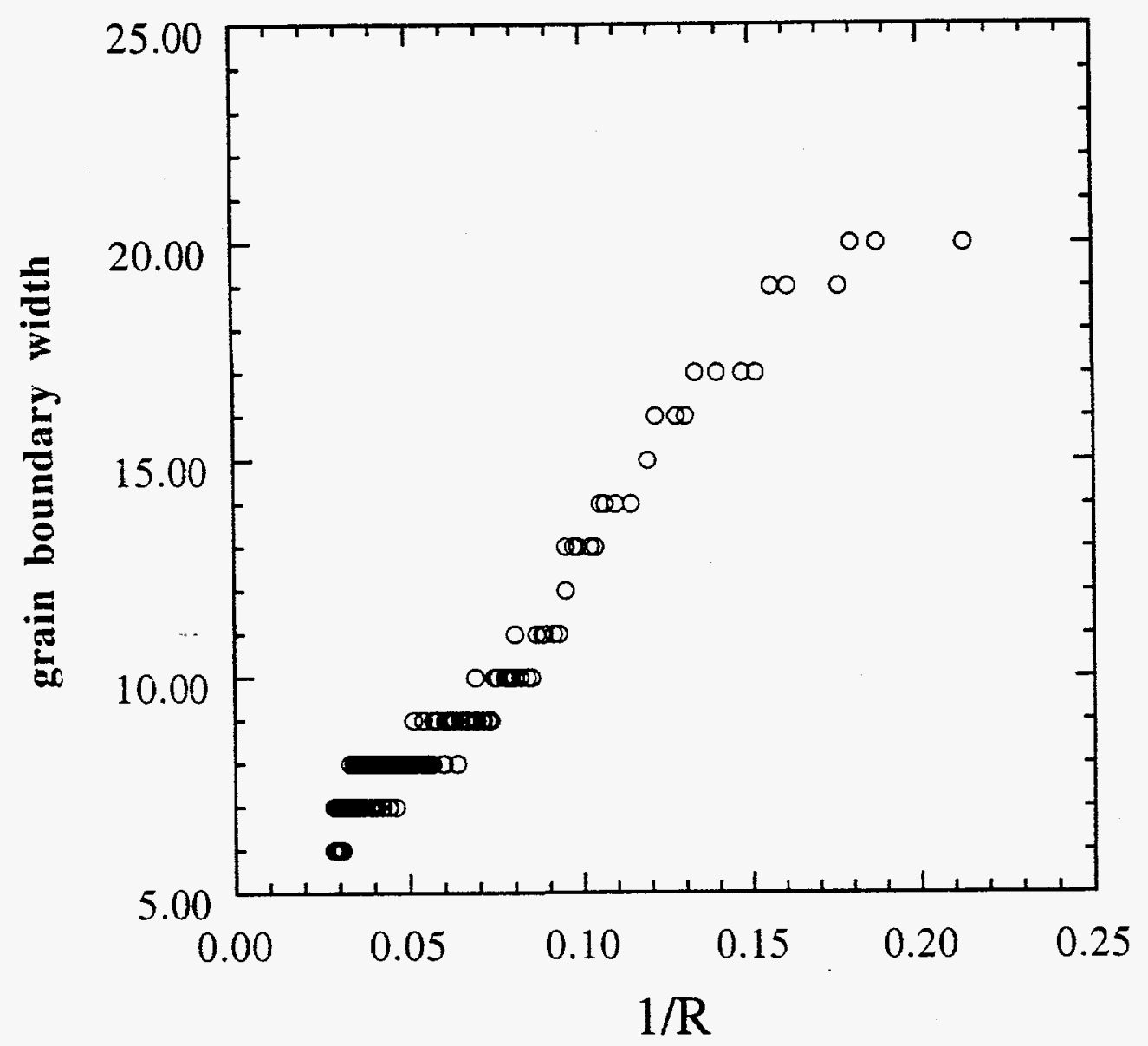

Fig. 7. The calculated dependence of grain boundary width on the mean curvature of a circular grain. 


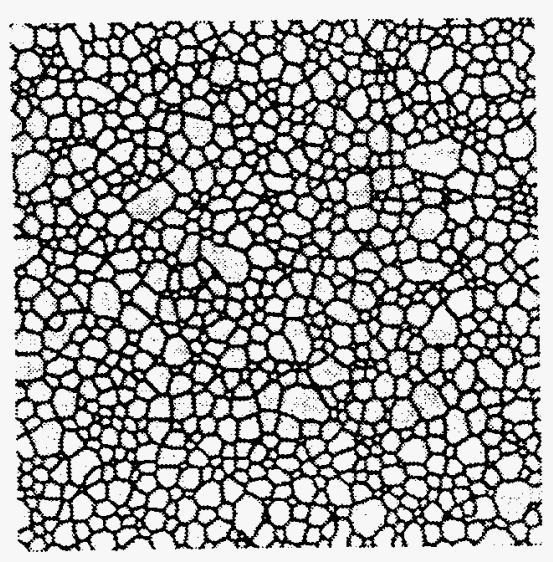

(a)

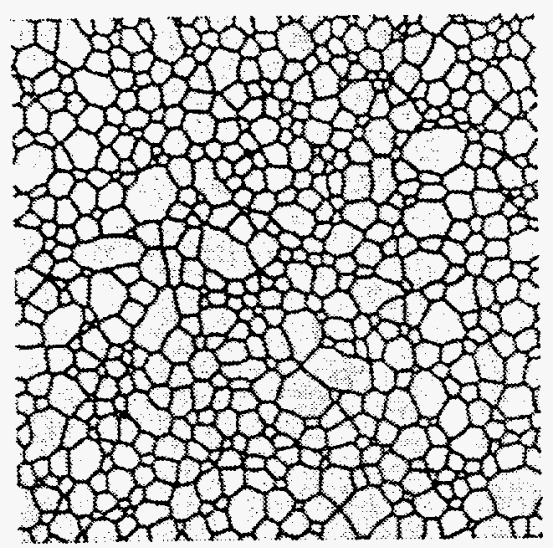

(c)

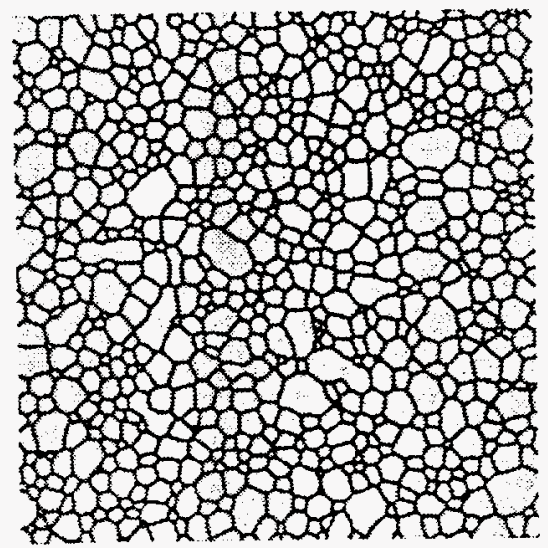

(b)

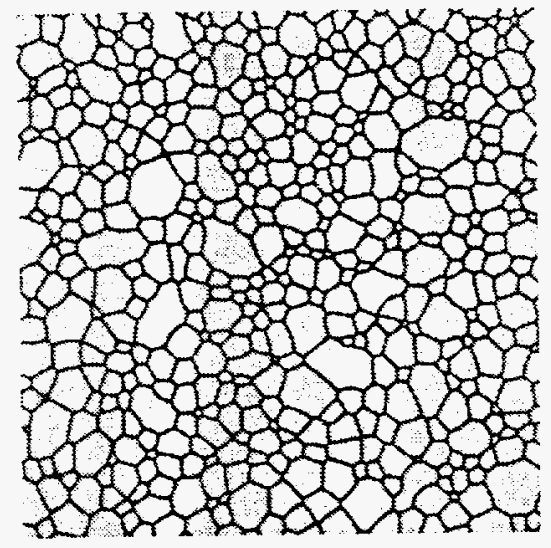

(d)

Fig. 8. The microstructural evolution of grain growth with solute-drag in a 2-D polycrystalline system. (a) $\mathrm{t}=5000 ;$ (b) $\mathrm{t}=10000 ;$ (c) $\mathrm{t}=15000 ;$ (d) $\mathrm{t}=20000$. 


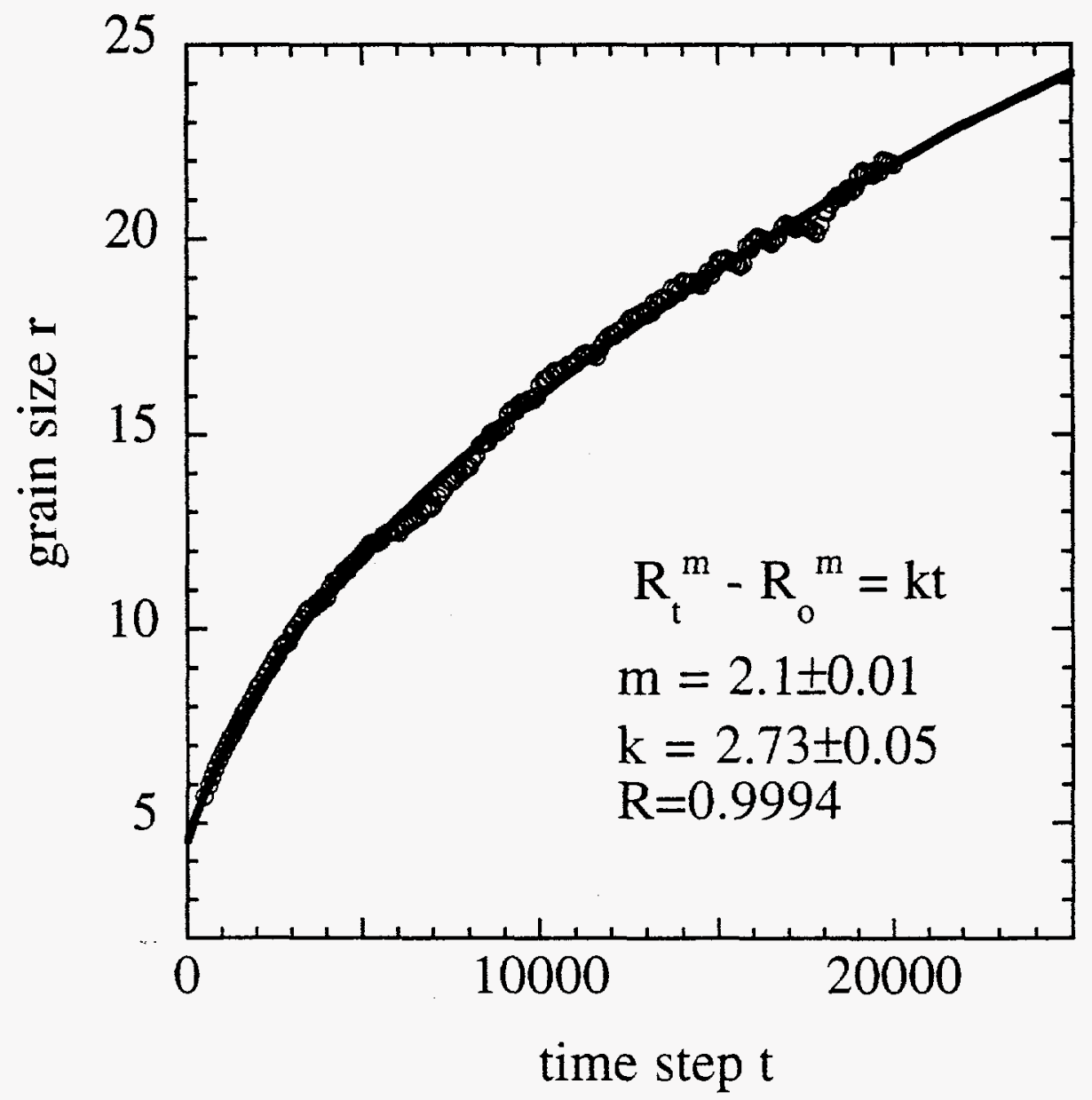

Fig. 9. The time dependence of average grain size under kinetic condition $D / L=1.0$. The solid line is a nonlinear fit to power growth law $R_{t}{ }^{m}-R_{o}{ }^{m}=k t$, which gives $\mathrm{m}=2.1 \pm 0.01$. 


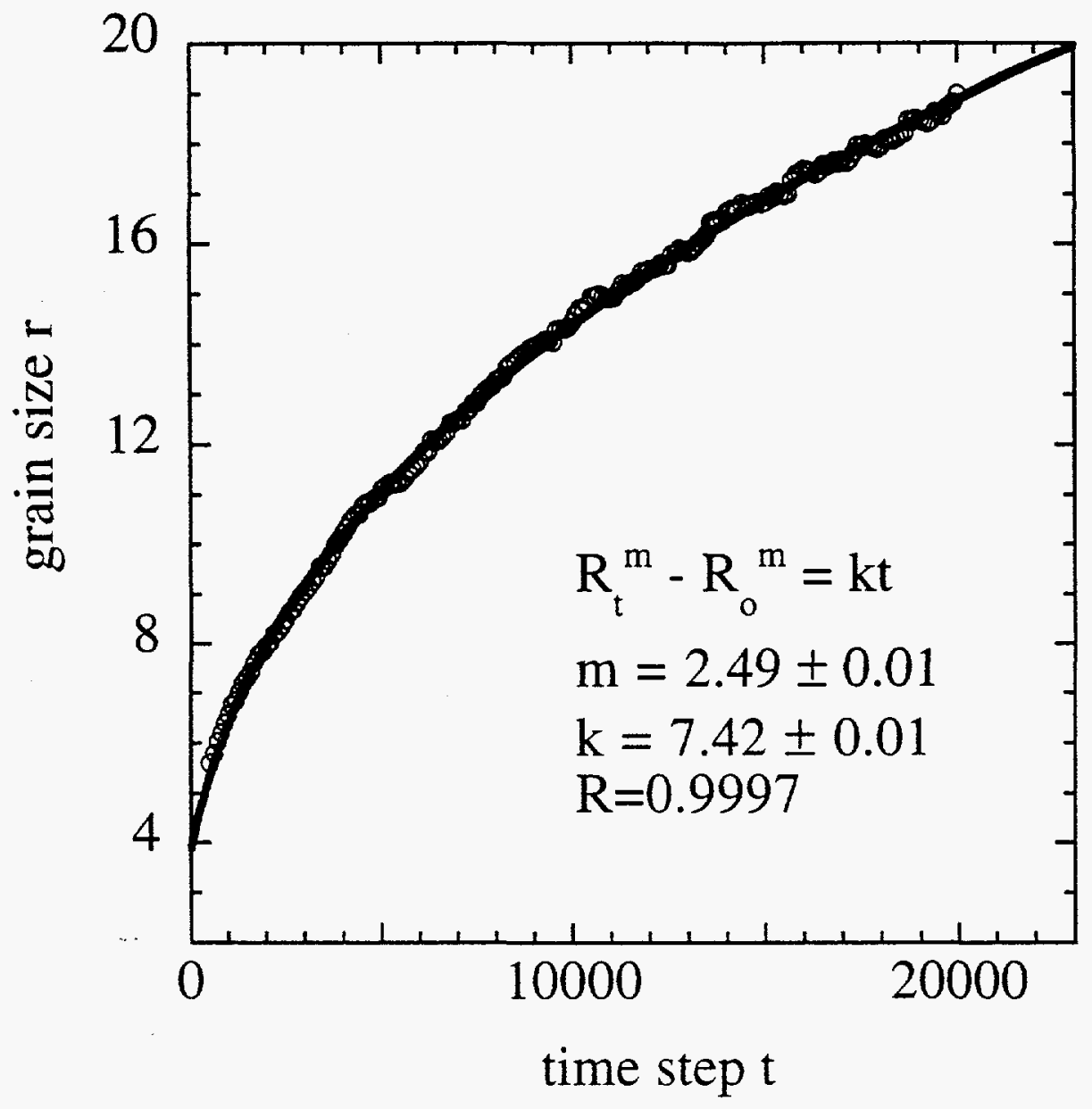

Fig. 10. The time dependence of average grain size under kinetic condition $D / L=0.5$. The solid line is a nonlinear fit to power growth law $R_{t}^{m}-R_{o}^{m}=k t$, which gives $m=2.49 \pm 0.01$. 


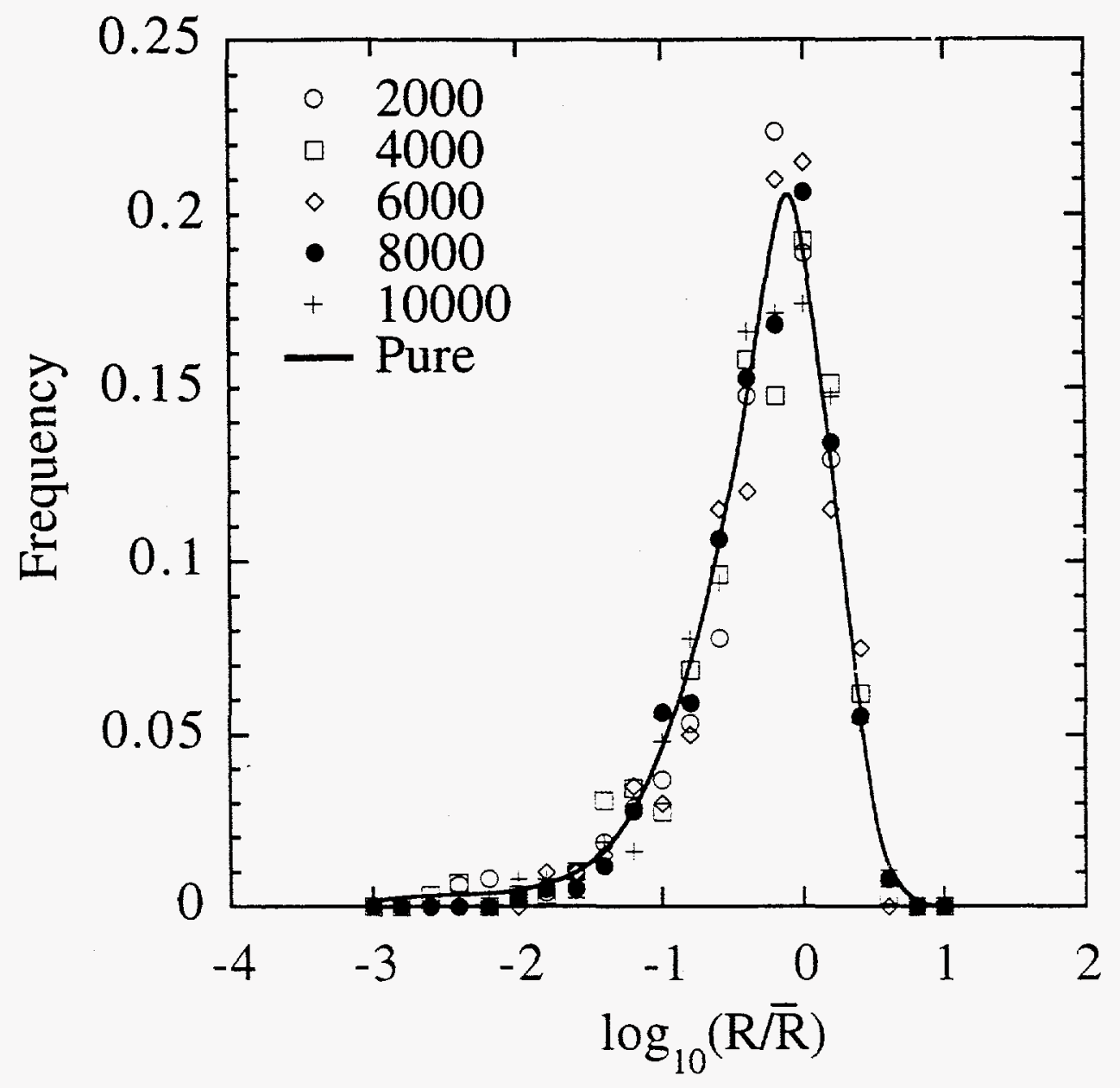

Fig. 11 The time dependence of grain size distributions under kinetic condition $\mathrm{D} / \mathrm{L}=1.0$. The solid line is a typical grain size distribution in a pure svstem (without solute-drag). 


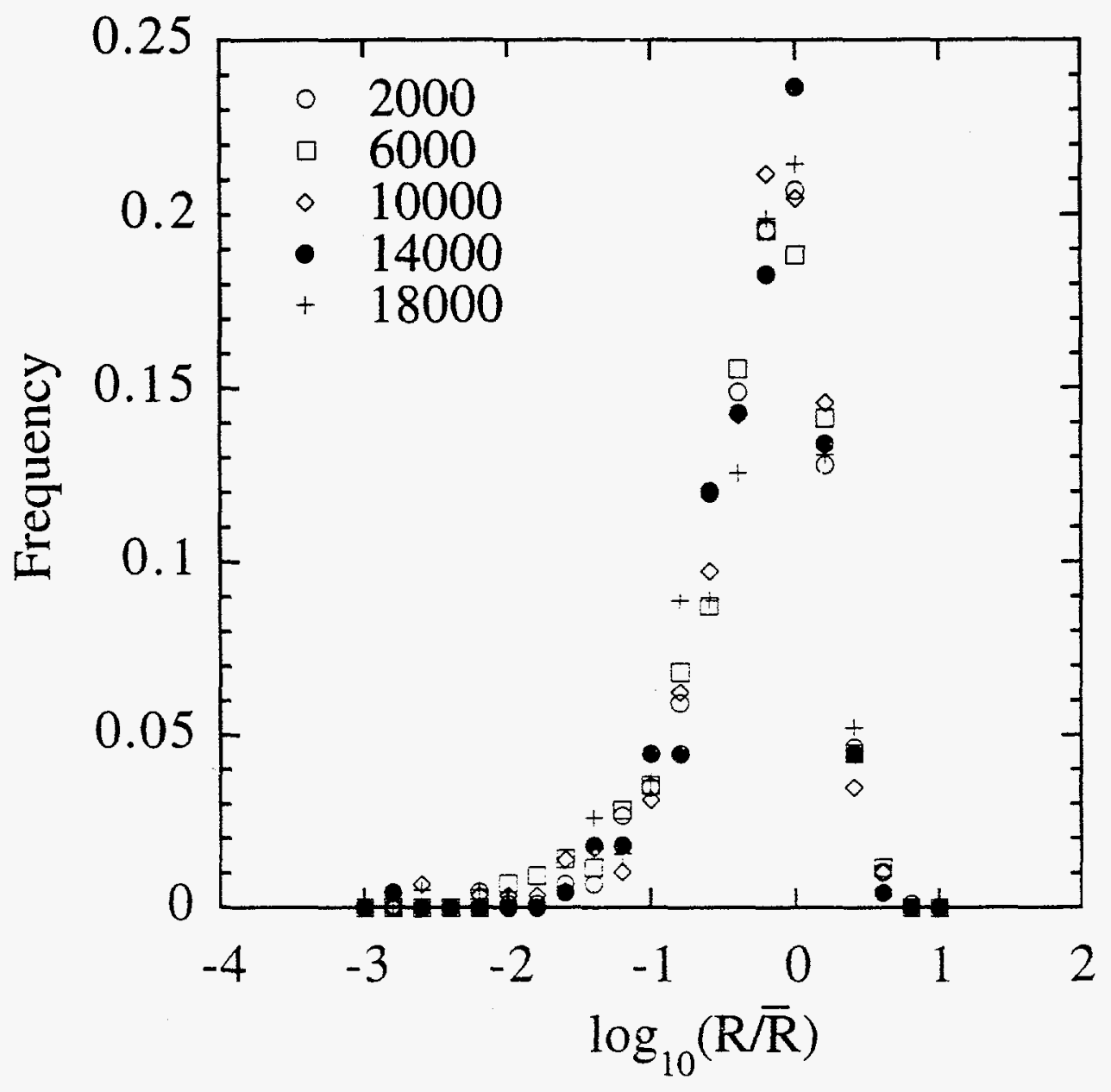

Fig. 12. The time dependence of grain size distributions under kinetic condition $D / L=0.5$. 


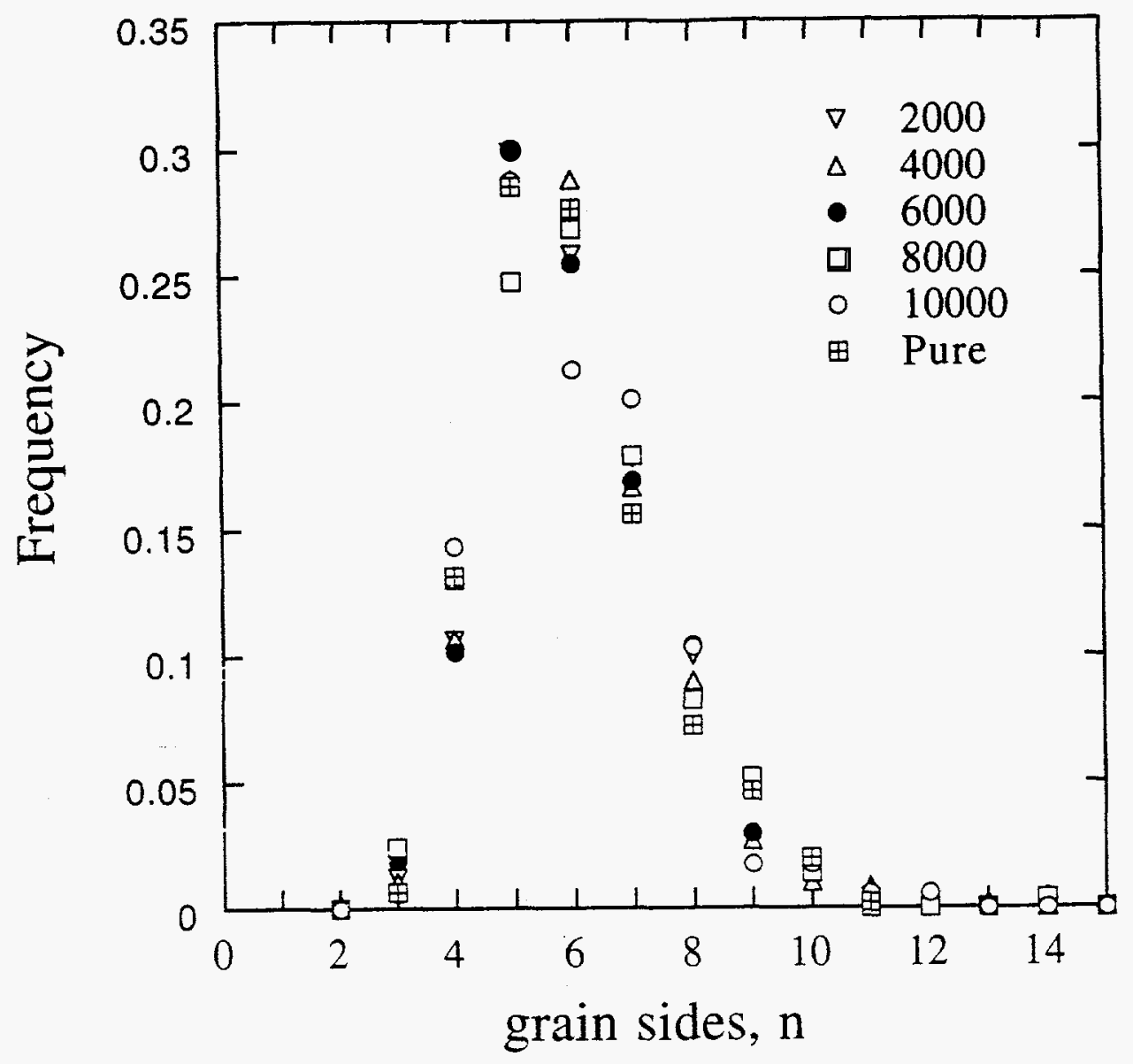

Fig. 13, The time dependence of grain topological distributions under kinetic condition $\mathrm{D} / \mathrm{L}=1.0$. A topological distribution for a pure system (without solute-drag) is shown in this figure for comparison. 


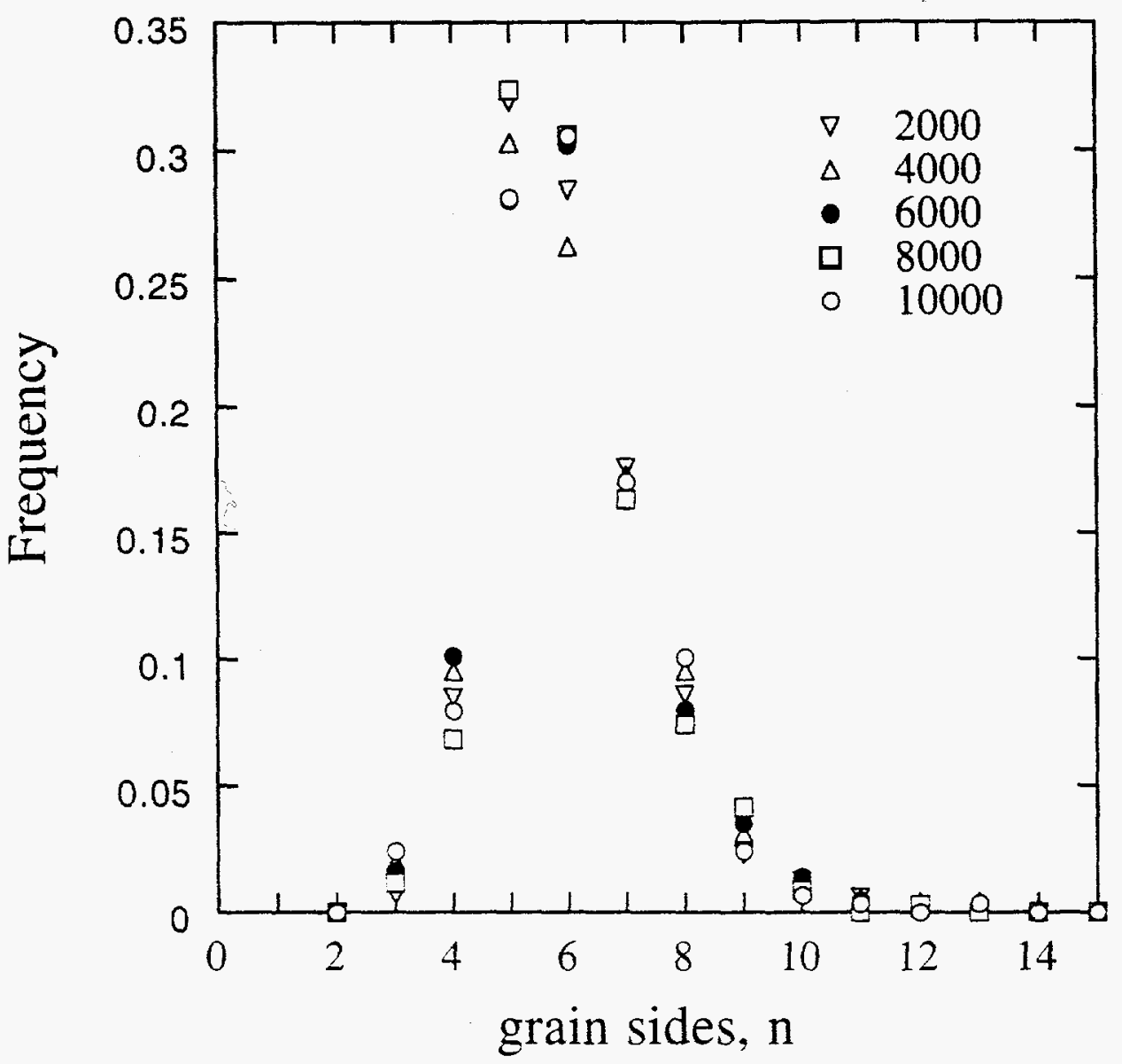

Fig. 14, The time dependence of grain topological distributions under kinetic condition $\mathrm{D} / \mathrm{L}=0.5$. 